\title{
Fe azaphthalocyanine unimolecular layers (Fe AzULs) on carbon nanotubes for realizing highly active oxygen reduction reaction (ORR) catalytic electrodes
}

\author{
Hiroya Abe $\mathbb{E}^{1,2}$, Yutaro Hirai ${ }^{3}$, Susumu Ikeda', Yasutaka Matsuo ${ }^{4}$, Haruyuki Matsuyama ${ }^{5}$, Jun Nakamura $\mathbb{0}^{5}$, \\ Tomokazu Matsue ${ }^{6}$ and Hiroshi Yabu ${ }^{1}$
}

\begin{abstract}
A new class of Pt-free catalysts was designed that included molecular iron phthalocyanine (FePc) derivatives, namely, iron azaphthalocyanine (FeAzPc) unimolecular layers (Fe AzULs) adsorbed on oxidized multiwall carbon nanotubes (oxMWCNTs). FeAzPcs were dissolved in organic solvents such as dimethyl sulfoxide (DMSO), and catalytic electrodes modified with molecularly adsorbed FeAzPcs were successfully prepared. The optimized composition of the catalytic electrodes was determined, and the electrodes exhibited superior activity for the oxygen reduction reaction (ORR) and better durability than conventional FePc catalytic electrodes and commercial Pt/C due to the electron-withdrawing properties of the pyridinic nitrogen in FeAzPcs. The catalytic electrodes that were molecularly modified with FeAzPcs have higher activities than those composed of FeAzPc crystals and oxMWCNTs. To the best of our knowledge, among all of the conventional catalysts based on modified MWCNTs and oxMWCNTs, this catalyst exhibits the highest activity. Unlike other Pt-free catalytic electrodes, the Fe AzUL catalytic electrodes can be prepared by low-cost processing without pyrolysis and are therefore promising catalytic electrode materials for applications, such as polymer electrolyte fuel cells and metal-air batteries.
\end{abstract}

\section{Introduction}

Pt-free catalysts with high catalytic activity are in demand as alternative materials to Pt supported on carbon black $(\mathrm{Pt} / \mathrm{C})$ to realize efficient, highly stable, and low-cost polyelectrolyte fuel cells ${ }^{1,2}$ and metal-air batteries $^{3,4}$. High oxygen reduction reaction (ORR) activity at the cathode contributes to the realization of such highenergy-density batteries because they promote low

\footnotetext{
Correspondence: Hiroya Abe (hiroya.abe.c4@tohoku.ac.jp) or

Tomokazu Matsue (matsue@tohoku.ac.jp) or

Hiroshi Yabu (hiroshi.yabu.d5@tohoku.ac.jp)

'WPI-Advanced Institute for Materials Research, Tohoku University, 2-1-1,

Katahira, Aoba-ku, Sendai 980-8577, Japan

${ }^{2}$ Frontier Research Institute for Interdisciplinary Sciences, Tohoku University, 6-

3, Aramaki-aza Aoba, Aoba-ku, Sendai 980-8578, Japan

Full list of author information is available at the end of the article.

These authors contributed equally: Hiroya Abe, Yutaro Hirai
}

overpotentials and four-electron reactions. $\mathrm{Pt} / \mathrm{C}$ has been most widely used as a cathode to achieve high ORR activity in fuel cells due to the high catalytic activity on the Pt surfaces ${ }^{5}$. However, the high cost and low durability of Pt have prevented its widespread application in fuel cells and air-metal batteries ${ }^{6}$.

Many studies have attempted to fabricate nonprecious metal catalysts consisting of carbon materials doped with heteroatoms, including iron, cobalt, nitrogen, and sulfur ${ }^{7-13}$, as alternatives to Pt-based catalysts. Based on these previous studies of heteroatom-doped carbon materials, $\mathrm{MN}_{4}$ complexes, which are composed of metal atoms with four coordinated pyridinic nitrogen ligands, show high affinity for oxygen molecules and catalytic activity for the $\mathrm{ORR}^{14-17}$ at their central metal. 
(a)

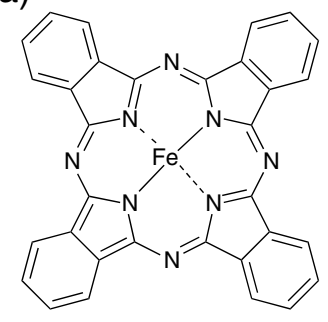

(c)

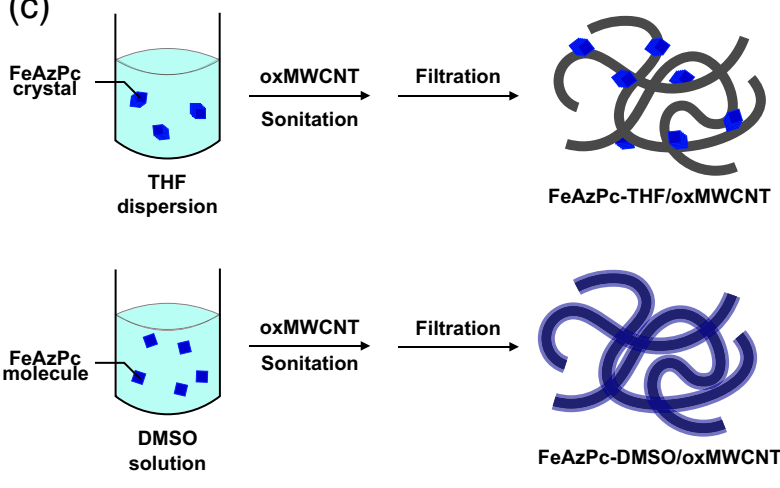

Fig. 1 Chemical structures of $\mathbf{a}$ iron phthalocyanine and $\mathbf{b}$ iron-tetra2,3-pyridinoporphyrazine (FeAzPc-4N). c Schematic image of a carbon nanotube with molecularly adsorbed FeAzPc-4N

Macrocyclic molecules with an $\mathrm{MN}_{4}$ structure have also been examined for use as ORR electrocatalysts ${ }^{18-23}$. For example, composite electrodes composed of nanocrystalline iron phthalocyanine (FePc, Fig. 1a) and carbon nanotubes (CNTs) also exhibit good ORR activity. FePc on graphene oxide (GO) exhibits excellent ORR activity comparable to that of $\mathrm{Pt} / \mathrm{C}^{24}$. This FePc/GO catalyst can be prepared via simple mixing of $\mathrm{GO}$ and FePc dispersions without the need for high-temperature or highpurity gas treatment. Recently, molecularly adsorbed FePc supported on pyridine-functionalized $\mathrm{CNTs}^{22}$ exhibited higher ORR activity than FePc/GO because the pyridine groups on the CNTs act as anchors for the FePc molecules through the Fe center via FePc-pyridinic N coordination, and the FePc molecules are immobilized in the single-molecule state. It was suggested that the singlemolecule adsorption of FePc on carbon materials prevented the low conductivities of the crystalline state and increased the catalytic activity of FePc due to $\pi-\pi$ interactions. However, these studies focus only on FePc; thus other macrocyclic catalytic molecules have not yet been examined in detail.

Furthermore, although solution processing is normally suitable for single-molecule adsorption of FePc molecules onto carbon materials and control of the amount of catalyst loading, elaborate solid-state grinding has usually been employed to form catalytic electrodes due to the low solubility of FePc. Since a small amount of FePc can be dissolved in some organic solvents, a few approaches have been reported to immobilize molecular FePcs onto chemically modified carbon materials with high-temperature refluxing of the $\mathrm{FePc}$ solution $^{24}$; however, roomtemperature processing to immobilize FePcs onto carbon materials to achieve a catalytic activity high enough for the material to serve as an alternative to $\mathrm{Pt} / \mathrm{C}$ has not been realized. Recently, we found that azaphthalocyanine (AzPc) derivatives are well dissolved in dimethyl sulfoxide (DMSO) and other polar organic solvents ${ }^{25}$. Furthermore, improvement of the catalytic activity is also expected owing to the electron-withdrawing properties of introduced $\mathrm{N}$ atoms because the energy level difference between the catalyst molecule and oxygen molecules decreases $^{26}$. Therefore, we focused on Fe azaphthalocyanines (FeAzPcs), where four pyridine rings are substituted for the four benzene rings of FePc to increase the solubility in organic solvent and the catalytic activities relative to those of FePc due to the polarity and electronwithdrawing properties of the pyridine and pyrazine rings. Herein, we present the formation of catalytic electrodes of FeAzPcs, including molecular layers of iron-tetra-2,3pyridinoporphyrazine (FeAzPc-4N, Fig. 1b) supported on oxidized multiwall carbon nanotubes (oxMWCNTs) by solution processing. The ORR activity performances of these azaphthalocyanine unimolecular layers (AzULs) on oxMWCNTs under alkaline conditions were evaluated (Fig. 1c).

\section{Results and discussion}

\section{Synthesis and characterization of FeAzPcs}

FeAzPc-4N was synthesized from 2,3-dicyanopyridine, $\mathrm{FeCl}_{3} \cdot 6 \mathrm{H}_{2} \mathrm{O}$, and 1,8-diazabicyclo[5.4.0] undec-7-ene (DBU) in a mixture of methanol and DMSO under $\mathrm{N}_{2}$ bubbling (Scheme S1). The yield of FeAzPc- $4 \mathrm{~N}$ was $36 \%$. The electrospray ionization mass spectrometry (ESI-MS) results (Fig. S1) showed the main peaks of FePc and FeAzPc-4N at $m / z=568$ and 572 . Some peaks that appeared around the main peak mainly originated from natural isotopes of Fe. These peaks were well matched to the theoretical molecular weight of the respective materials. Ultraviolet-visible (UV-Vis) spectra (Fig. S2) for FePc and FeAzPc-4N dissolved in DMSO showed the Q band, which is a characteristic absorbance peak of phthalocyanine derivatives, at 655 and $627 \mathrm{~nm}$, respectively. The calculated band gaps of FePc and FeAzPc-4N were 1.89 and $1.97 \mathrm{eV}$, respectively. The absorption peak of the $\mathrm{Q}$ band was blueshifted with an increase in the number of $\mathrm{N}$ atoms in the FePc derivatives. $\mathrm{N}$ is more electronegative than $\mathrm{C}$, and FeAzPc-4N exhibits lower degeneracy than FePcs; therefore, the absorption peaks of the FeAzPcs are blueshifted. This trend of band gaps is in good agreement with previous reports ${ }^{27}$. 
Table 1 Solubility of iron phthalocyanine analogs

\begin{tabular}{llll}
\hline & DMSO & DMF & THF \\
\hline FePC & $0.45 \mathrm{~g} / \mathrm{L}$ & $0.45 \mathrm{~g} / \mathrm{L}$ & $0.049 \mathrm{~g} / \mathrm{L}$ \\
FeAzPC-4N & $0.80 \mathrm{~g} / \mathrm{L}$ & $0.46 \mathrm{~g} / \mathrm{L}$ & $0.018 \mathrm{~g} / \mathrm{L}$ \\
\hline
\end{tabular}

DMF dimethyl formamide, DMSO dimethyl sulfoxide, FePc iron phthalocyanine, FeAzPc- $4 N$ iron-tetra-2,3-pyridinoporphyrazine, $T H F$ tetrahydrofuran

The solubilities of FePc and FeAzPc-4N in DMSO, dimethyl formamide (DMF), and tetrahydrofuran (THF) are summarized in Table 1. The solubilities of FePc and FeAzPcs in DMSO were higher than those in THF and DMF. FeAzPcs showed higher solubility than FePc because of the introduced pyridine rings, which have higher polarity than benzene rings. From these results, DMSO solutions were used in this study for molecular adsorption onto oxMWCNTs.

FeAzPc-4N-DMSO/oxMWCNT electrodes were prepared by mixing a $0.1 \mathrm{mg} / \mathrm{mL}$ FeAzPc-4N DMSO solution and $30 \mathrm{mg}$ oxMWCNTs with various amounts of solution from $15 \mathrm{~mL}$ to $120 \mathrm{~mL}$. The resulting solution was filtered to collect, and the samples were washed three times each with methanol and chloroform. Finally, FeAzPc-4NDMSO/oxMWCNT samples with an FeAzPc-4N concentration of 5-40 wt\% were prepared. To compare the effect of solvents used for modification on the ORR activities, composites of FeAzPc and oxMWCNTs were also prepared in $\mathrm{THF}^{23}$ and are denoted as FeAzPc-4NTHF/oxMWCNT_50 wt\%. FePc-DMSO/oxMWCNT_20 wt\% and FePc-THF/oxMWCNT_50 wt\% were also synthesized by the same procedure used for the FeAzPc/ oxMWCNT catalysts. The contents of FePc derivatives relative to oxMWCNTs are shown in Table S1.

\section{Characterization of oxMWCNTs modified with FeAzPcs}

Transmission electron microscopic (TEM) images of FeAzPc-4N-THF/oxMWCNT_50 wt\% and FeAzPc-4NDMSO/oxMWCNT_20 wt\% are shown in Fig. 2a, b, respectively. Small particles (approximately $100 \mathrm{~nm}$ ) were observed in both FeAzPc-4N-THF/oxMWCNT_50 wt\% and FePc-THF/oxMWCNT_50 wt\% (Fig. S3). X-ray diffraction (XRD) pattern of FeAzPc-4N-THF/ oxMWCNT_50 wt\% shows peaks derived from pristine FeAzPc-4N crystals and oxMWCNTs (Fig. 2c). A small number of peaks from FeAzPc-4N crystals were observed in the FeAzPc-4N-DMSO/oxMWCNT_20 wt\% pattern (Fig. 2c), which implies immature crystal formation. The peak at $2 \theta=25.6^{\circ}$ is derived from the $\mathrm{Si}$ substrate. Asprepared FeAzPc-4N had some sharp peaks at mainly $2 \theta$ $=8.8^{\circ}, 10.8^{\circ}$, and $22-24^{\circ}$, which indicate that FeAzPc-4N forms a crystal structure. Similarly, the XRD pattern of FeAzPc-4N-THF/oxMWCNT involved peaks at the same $2 \theta$ value; therefore, FeAzPc-4N crystals were also present in FeAzPc-4N-THF/oxMWCNT. In contrast, the XRD pattern of FeAzPc-4N-DMSO/MWCNT had no peaks in common with that of as-prepared FeAzPc-4N, which indicates that FeAzPc-4N was present only as single molecules or clusters of a few molecules. As seen from the XRD characterizations, FeAzPc-4N was present in the FeAzPc-4N-THF/oxMWCNT and FeAzPc-4N-DMSO/ oxMWCNT samples as crystals and as groups of a few molecules, respectively.

To confirm the presence of FeAzPc-4N on the surface of oxMWCNTs, X-ray photoelectron spectroscopy (XPS) measurements were performed (Fig. S4). The XPS spectra of FeAzPc-4N-DMSO/oxMWCNT showed a separation peaks of the Fe 2p core level at 708 and $722 \mathrm{eV}$, which were assigned as $\mathrm{Fe} 2 \mathrm{p}_{3 / 2}$ and $\mathrm{Fe} 2 \mathrm{p}_{1 / 2}$, respectively. Similarly, the $\mathrm{N}$ 1s peak for FeAzPc-4N-DMSO/ oxMWCNT was observed at $399 \mathrm{eV}$. In contrast, the Fe $2 \mathrm{p}$ and $\mathrm{N}$ 1s peaks of oxMWCNTs were not observed because FeAzPc-4N was only present in FeAzPc-4NDMSO/oxMWCNTs. The peak intensities of Fe 2p and $\mathrm{N}$ $1 \mathrm{~s}$ increased with the amount of FeAzPc. The amounts of FeAzPc-4N in the catalysts were calculated from the ratio between the $\mathrm{C} 1 \mathrm{~s}$ and $\mathrm{N}$ 1s peaks (Table S1). From the XPS analysis, the amounts of FePc and FeAzPc-4N in the samples (FePc-DMSO/oxMWCNT_20wt\% and FeAzPc4N-DMSO/oxMWCNT_20wt\%) were 8.5 and $8.3 \mathrm{wt} \%$, respectively, indicating that the introduction of the pyridinic rings in place of the benzene rings did not affect the amount of that molecule. The FeAzPc-4N content was also determined using thermogravimetric-differential thermal analysis (TG-DTA; Figs. S5 and S6). As seen from the TG-DTA results for pristine oxMWCNTs and FeAzPc-4N, decomposition occurred at approximately $420-600{ }^{\circ} \mathrm{C}$ and at approximately $350-380^{\circ} \mathrm{C}$, respectively.

To confirm the molecular modification of FeAzPc- $4 \mathrm{~N}$ on the oxMWCNTs, the samples were observed using high-angle annular dark field scanning transmission electron microscopy (HAADF-STEM) and electron energy loss spectroscopy (EELS). Figure $2 \mathrm{~d}$ shows an HAADF-STEM image of the FeAzPc-4N-DMSO/ oxMWCNT_20 wt\% electrode. From this image, the multiwalled structure of the oxMWCNTs is clearly evident. Small bright spots were also observed on the surface of oxMWCNTs. Figure 2e shows a close-up HAADFSTEM image of the FeAzPc-4N-DMSO/oxMWCNT_20 wt\% electrode. EELS spectra were measured at each pixel shown in Fig. 2e. From the EELS spectra obtained from the surface of the electrode, peaks attributed to Fe and $\mathrm{N}$ were observed at these positions (each size was $0.5 \mathrm{~nm}^{2}$ ) close to the bright spots (Fig. 2f). The molecular size of phthalocyanines is approximately $1.7 \mathrm{~nm}^{28}$; therefore, the EELS analysis provides evidence for the molecular adsorption of FeAzPc-4N onto oxMWCNTs. 
(a)

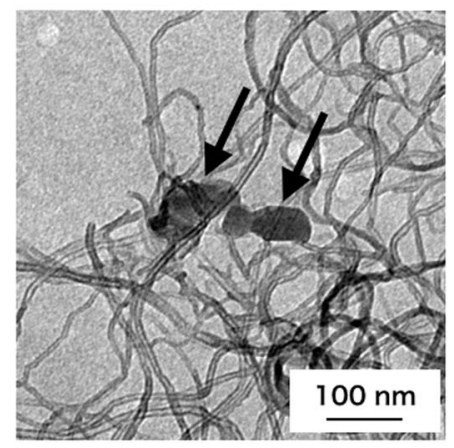

(d)

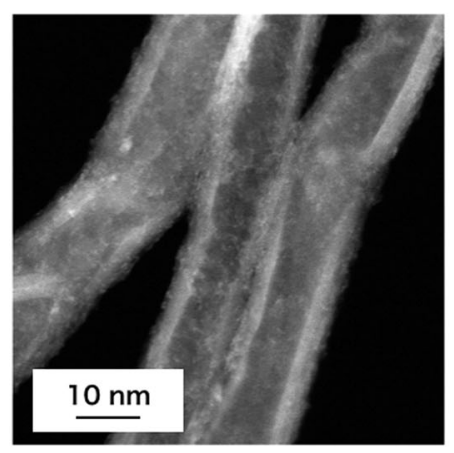

(b)

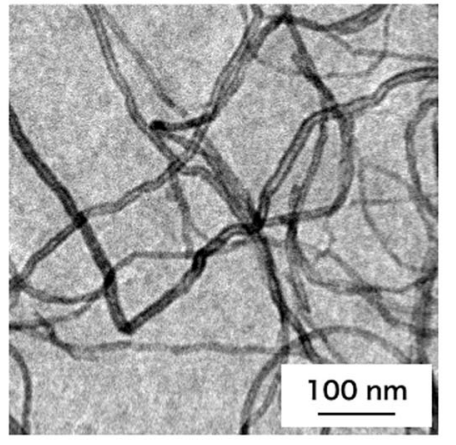

(e)

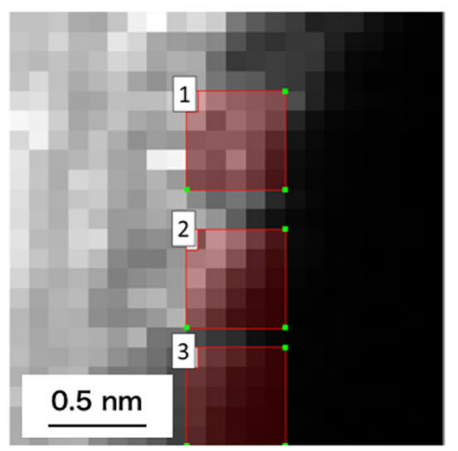

(c)

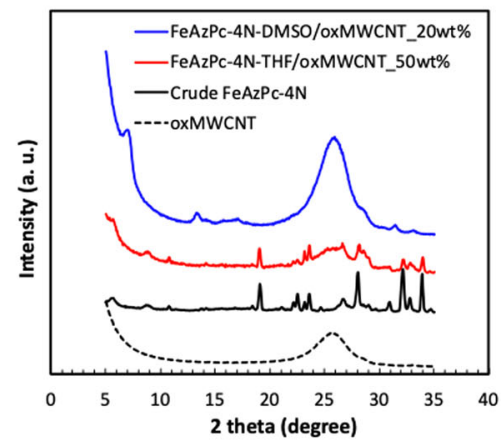

(f)

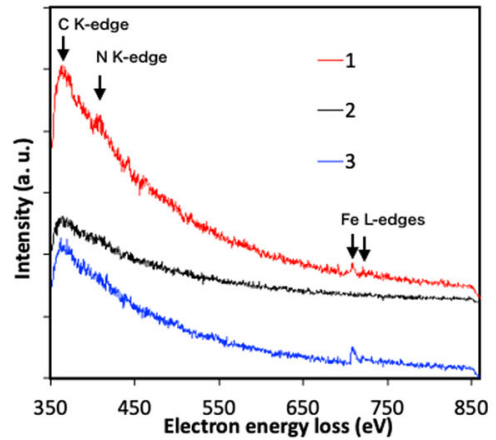

Fig. 2 Transmission electron microscopic image of a FeAzPc-4N-THF/oxMWCNT_50 wt\% and b FeAzPc-4N-DMSO/oxMWCNT_20 wt\%. c X-ray diffraction patterns of oxidized multiwall carbon nanotubes (oxMWCNTs; black dashed line), FeAzPc-4N-THF/oxMWCNT_50 wt\% (black solid line), FeAzPc-4N-DMSO/oxMWCNT_20 wt\% (red solid line), and FeAzPc-4N crude (red dashed line). d, e High-angle annular dark field scanning transmission electron microscopic images of FeAzPc-4N-DMSO/oxMWCNT_20 wt\%. f Electron energy loss spectroscopic spectrum of FeAzPc-4NDMSO/oxMWCNT_20 wt\%. The spectra of each area in $\mathbf{e}$ are shown in $\mathbf{f}$

\section{ORR activities of the prepared catalysts}

The differences in the ORR activities of the catalytic electrodes prepared from THF and DMSO solutions of FeAzPc-4N are discussed first. Figure 3a shows the linear sweep voltammetric (LSV) curves of FeAzPc-4N-THF/ oxMWCNT_50 wt\% (black line) and FeAzPc-4N-DMSO/ oxMWCNT_20 wt\% (red line) measured in an $\mathrm{O}_{2}$-saturated $0.1 \mathrm{M} \mathrm{KOH}$ solution. The onset potentials $\left(E_{\text {onset }}\right)$ of FeAzPc-4N-THF/oxMWCNT and FeAzPc-4N-DMSO/ oxMWCNT were 1.00 and $1.04 \mathrm{~V}$ vs. reversible hydrogen electrode (RHE), respectively, and the respective half wave potentials $\left(E_{1 / 2}\right)$ were 0.898 and $0.913 \mathrm{~V}$ vs. RHE. The ORR activity of the FeAzPc-4N-DMSO/oxMWCNT_20 wt\% electrode was higher than that of the FeAzPc-4NTHF/oxMWCNT_50 wt\% electrode, even though the catalyst content was much lower. $E_{1 / 2}$ for the FePc-THF/ oxMWCNT and FePc-DMSO/oxMWCNT electrodes were 0.871 and $0.881 \mathrm{~V}$ vs. RHE (Fig. S7), respectively, which was the same tendency as that for the FeAzPc-4Nbased electrodes. These results indicate that the molecular adsorption of catalytic molecules is crucial for the promotion of ORR activity.
It is well known that a small amount of Fe contamination exists in commercially available CNTs. To avoid this effect, we used mainly oxMWCNTs, which were chemically oxidized under strong acidic conditions, which removes $\mathrm{Fe}$ atoms from the oxMWCNTs. The removal of Fe was proven by the XPS results shown in Fig. S4, in which no Fe peak was observed. Moreover, oxMWCNT itself did not show any significant ORR activity, as shown in Fig. 3b. From these results, the contaminated Fe did not exist in the sample, and it had no effect on the ORR activities of oxMWCNTs. As shown in Fig. S9, since the ORR activities of FeAzPc-4Nmodified oxMWCNTs and MWCNTs were identical, there was also no effect of the trace of Fe-containing commercially available MWCNTs on ORR activity. The relationship between the ORR activities and the FeAzPc-4N contents is shown in Fig. S8. The ORR activity increased with the FeAzPc-4N content up to $20 \mathrm{wt} \%$, where the best ORR activity was observed. In the case of $40 \mathrm{wt} \%$, both $E_{\text {onset }}$ and $E_{1 / 2}$ decreased compared with that for $20 \mathrm{wt} \%$ FeAzPc-4N. This phenomenon is discussed later.

To investigate the effect of oxidized groups in oxMWCNTs on the ORR activity, modification of 


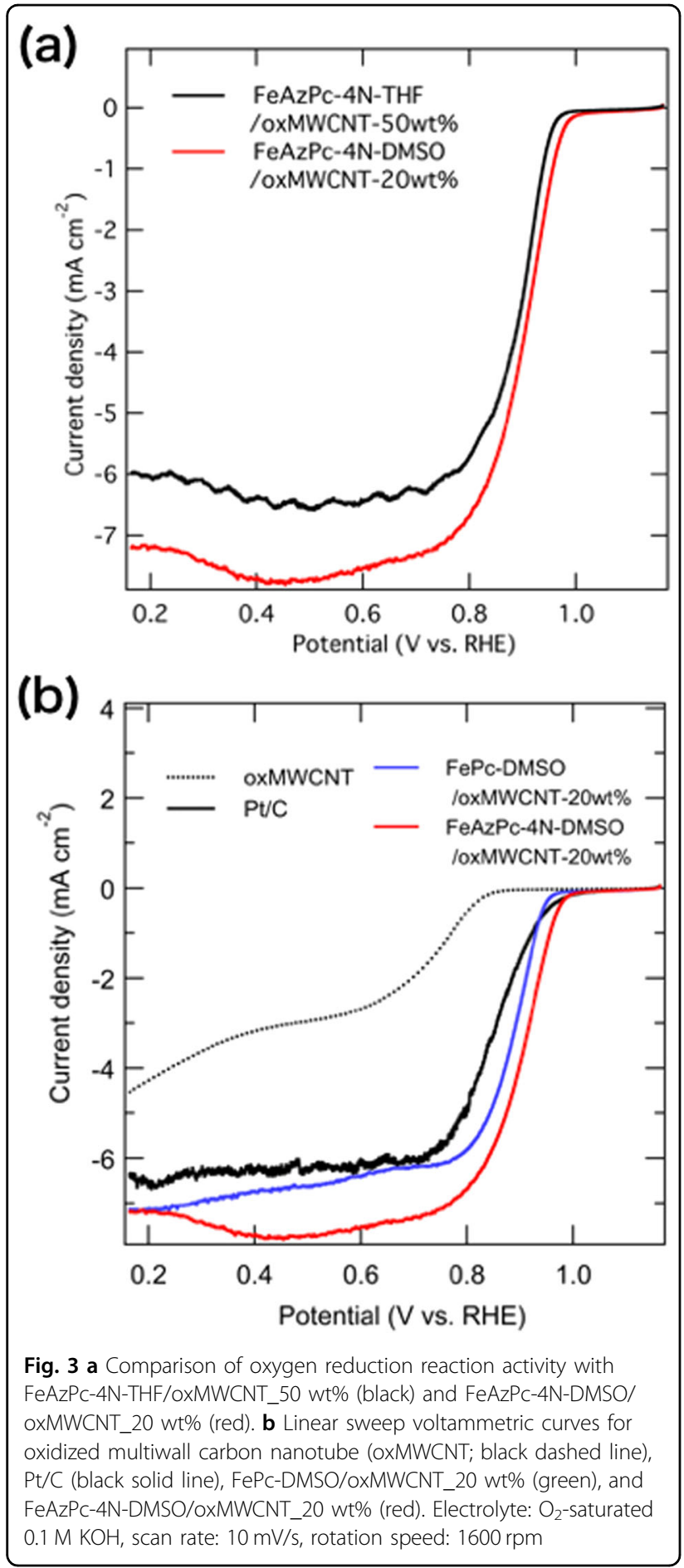

FeAzPc-4N on MWCNTs without oxidation (FeAzPc4N-DMSO/MWCNT) was also evaluated (Fig. S9). $E_{\text {onset }}$ and $E_{1 / 2}$ for FeAzPc-4N-DMSO/MWCNT were 1.00 and $0.913 \mathrm{~V}$ vs. RHE, respectively, which were identical to the values for the oxMWCNT-based electrode. This result indicates that the oxidized groups of oxMWCNTs did not have an effect on the ORR activity. Figure 3b shows LSV curves for oxMWCNT, FePc-DMSO/oxMWCNT_20 wt \%, FeAzPc-4N-DMSO/oxMWCNT_20 wt\%, and Pt/C. The ORR activities of these catalysts are summarized in Table 2. From the LSV curve for the oxMWCNT electrode, the catalytic activity was very low. The $\mathrm{Pt} / \mathrm{C}$ electrode (black line) showed high $E_{\text {onset }}$ and $E_{1 / 2}$ values. The FeAzPc derivatives exhibited high $E_{\text {onset }}$, as with $\mathrm{Pt} / \mathrm{C}$, and had higher $E_{1 / 2}$ values than did FePc and Pt/C. In particular, the highest ORR activity was observed for FeAzPc4N-DMSO/oxMWCNT_20 wt\%, which was superior to representative carbon materials modified with conventional catalysts (Table S2).

\section{Catalytic activities of FeAzPc-4N-DMSO/oxMWCNT_20 wt\%}

To evaluate the ORR activity in detail, the ORR kinetics of the catalytic reactions were assessed in $0.1 \mathrm{M} \mathrm{KOH}$ using a rotating-disk electrode and an rotating ring-disk electrode (RRDE). Figure 4a, b shows LSV curves and Koutecky-Levich (K-L) plots for FeAzPc-4N-DMSO/ oxMWCNT_20 wt\%, respectively. The K-L plots are used to determine the kinetic parameters for the ORR, the details of which are given in the "Experimental" section. The linearity of the K-L plots shows the reaction kinetics for dissolved oxygen and the electron transfer numbers for the ORR at various potentials ${ }^{29}$. LSV curves and K-L plots for commercial Pt/C (Fig. S10) were measured. From these K-L plots at $0.7-0.8 \mathrm{~V}$, the calculated electron transfer numbers $(n)$ for the FeAzPc-4N-DMSO/ oxMWCNT_20 wt\% and commercial Pt/C catalysts were 3.7 and 4.1, respectively, which indicated that $\mathrm{O}_{2}$ was reduced on the catalysts through a direct four-electron reaction. The electron transfer number for each catalyst was also evaluated using $\mathrm{HO}_{2}{ }^{-}$yields measured with an RRDE in $0.1 \mathrm{M} \mathrm{KOH}$. The $\mathrm{HO}_{2}{ }^{-}$yields with FeAzPc-4NDMSO/oxMWCNT_20 wt\% (Fig. 4c, red) and commercial Pt/C (Fig. 4c, black) were $<6 \%$ and $<10 \%$, respectively. The electron transfer numbers of these catalysts were both approximately 3.9, as calculated using Eq. (3). This value was identical to the electron transfer numbers calculated from the K-L plots. These results indicate that each ORR reaction is a direct four-electron reaction, which contributes to the realization of high-powerdensity fuel cells and metal-air batteries.

The Tafel plots for FeAzPc-4N-DMSO/oxMWCNT_20 $w t \%$ (red) and $\mathrm{Pt} / \mathrm{C}$ (black) are shown in Fig. 4d. The slopes of the Tafel plots for FeAzPc-4N-DMSO/ oxMWCNT_20 wt\% and Pt/C at low overpotentials calculated using $J_{\mathrm{k}}$ were 29.1 and $61.8 \mathrm{mV}$ per decade, respectively. There are several limiting steps in the ORR, such as the protonation of superoxide $\left(\mathrm{O}_{2}{ }^{-}\right)$and the adsorption of $\mathrm{O}_{2}$ molecules on active sites ${ }^{30}$. The Tafel slope for FeAzPc-4N-DMSO/oxMWCNT_20 wt\% was much lower than that of the commercial $\mathrm{Pt} / \mathrm{C}$. The low Tafel slope indicates that the protonation of $\mathrm{O}_{2}{ }^{-}$on the 
Table 2 Summary of ORR activity

\begin{tabular}{llllll}
\hline Sample no. & Sample name & Surface-adsorbed FeAzPc ratio & $\boldsymbol{E}_{\text {onset }}$ & $\boldsymbol{E}_{\mathbf{1 / 2}}$ & Current density $^{\mathbf{b}}$ \\
\hline 1 & FeAzPc-4N-DMSO/oxMWCNT_20 wt\% & $8.3 \mathrm{wt} \%$ & $1.020 \mathrm{~V}$ & $0.913 \mathrm{~V}$ & $-7.71 \mathrm{~mA} / \mathrm{cm}^{2}$ \\
2 & FeAzPc-4N-THF/oxMWCNT_50 wt\% & $11.2 \mathrm{wt} \%$ & $1.000 \mathrm{~V}$ & $0.898 \mathrm{~V}$ & $-6.55 \mathrm{~mA} / \mathrm{cm}^{2}$ \\
3 & FePC-DMSO/OXMWCNT_20 wt\% & $8.5 \mathrm{wt} \%$ & $0.985 \mathrm{~V}$ & $0.881 \mathrm{~V}$ & $-6.62 \mathrm{~mA} / \mathrm{cm}^{2}$ \\
4 & FePc-THF/OXMWCNT_50 wt\% & $24.7 \mathrm{wt} \%$ & $0.980 \mathrm{~V}$ & $0.871 \mathrm{~V}$ & $-3.17 \mathrm{~mA} / \mathrm{cm}^{2}$ \\
5 & Pt/C & - & $1.035 \mathrm{~V}$ & $0.850 \mathrm{~V}$ & $-6.35 \mathrm{~mA} / \mathrm{cm}^{2}$ \\
6 & OXMWCNT & - & $0.90 \mathrm{~V}$ & $0.675 \mathrm{~V}$ & $-2.96 \mathrm{~mA} / \mathrm{cm}^{2}$ \\
\hline
\end{tabular}

DMF dimethyl formamide, DMSO dimethyl sulfoxide, FePC iron phthalocyanine, FeAzPc-4N iron-tetra-2,3-pyridinoporphyrazine, ORR oxygen reduction reaction, oxMWCNT oxidized multiwall carbon nanotube, THF tetrahydrofuran

${ }^{a}$ Surface-adsorbed FeAzPc ratio was measured by XPS

${ }^{\mathrm{b}}$ Current density at $0.5 \mathrm{~V}$

active sites of the catalyst is the rate-limiting step $^{29}$; therefore, the rate-limiting step of the ORR on FeAzPc$4 \mathrm{~N}-\mathrm{DMSO} / \mathrm{oxMWCNT} \_20 \mathrm{wt} \%$ was mainly the protonation of $\mathrm{O}_{2}{ }^{-}$on the active sites.

Figure 4e shows current-time chronoamperometric responses of the samples in $\mathrm{O}_{2}$-saturated $0.1 \mathrm{M} \mathrm{KOH}$ at $0.4 \mathrm{~V}$ vs. RHE for $45,000 \mathrm{~s}$ under stirring. The current value for $\mathrm{Pt} / \mathrm{C}$ decreased with an increase in the reaction time and dropped to $64 \%$ of the initial current after $45,000 \mathrm{~s}$. In contrast, the current value for FeAzPc-4NDMSO/oxMWCNT_20 wt\% remained almost constant at the initial current after $45,000 \mathrm{~s}$, and the final current was $98 \%$ of the initial current. Figure $4 \mathrm{f}$ shows LSV curves for FeAzPc-4N-DMSO/oxMWCNT_20 wt\% (red) and Pt/C (black) before (broken line) and after (solid line) the durability test. The $\Delta E_{1 / 2}$ values for these catalysts were 12 and $32 \mathrm{mV}$, respectively, which indicates that FeAzPc4N-DMSO/oxMWCNT_20 wt\% exhibits excellent durability compared with $\mathrm{Pt} / \mathrm{C}$.

The methanol tolerance of FeAzPc-4N-DMSO/ oxMWCNT_20 wt\% and Pt/C was also evaluated by LSV measured in $0.1 \mathrm{M} \mathrm{KOH}$ with $3 \mathrm{M}$ methanol (Fig. 4g). On the $\mathrm{Pt} / \mathrm{C}$ catalyst (black), an oxidation current was evident, which indicates that methanol oxidation occurred. In contrast, only slight changes were observed for FeAzPc-4N-DMSO/oxMWCNT_20 wt\% (red), which implies that FeAzPc-4N-DMSO/oxMWCNT_20 wt\% has better methanol tolerance than $\mathrm{Pt} / \mathrm{C}$.

\section{Activity evaluation for iron center}

The redox potential of $\mathrm{Fe}(\mathrm{III}) /(\mathrm{II})$ complexes in the $\mathrm{MN}_{4}$ macrocycle of $\mathrm{Fe}$ phthalocyanine derivatives is generally associated with ORR activity. The energy level of pyridinic nitrogen in carbon catalysts also decreases, which induces the adsorption of $\mathrm{O}_{2}$ molecules on carbon atoms of neighboring nitrogen atoms and gives results in ORR activities ${ }^{31}$. FeAzPc-4N has an $\mathrm{MN}_{4}$ macrocycle and pyridinic nitrogen moieties; therefore, the active center of FeAzPc-4N should be determined. The addition of $\mathrm{KCN}$ significantly reduces the activity of the $\mathrm{Fe}(\mathrm{III}) /(\mathrm{II})$ complexes in the $\mathrm{MN}_{4}$ macrocycle, and the catalytic activity of pyridinic nitrogen can thus be evaluated ${ }^{32}$. Figure $5 \mathrm{a}$ shows LSV curves for oxMWCNTs and FeAzPc-4NDMSO/oxMWCNT_20 wt\% before and after the addition of $10 \mathrm{mM} \mathrm{KCN}$. The ORR activity of the FeAzPc-4NDMSO/oxMWCNT after KCN addition decreased significantly and approached that for oxMWCNT, which suggested that the Fe ions in FeAzPc-4N mainly reacted with oxygen, while the pyridinic nitrogen moieties in FeAzPc-4N did not directly react with $\mathrm{O}_{2}{ }^{33}$. The ORR activity of KCN-treated FeAzPc-4N-DMSO/oxMWCNTs was recovered by simply rinsing the electrode with water to remove $\mathrm{CN}^{-}$ions absorbed on the active sites (Fig. S11).

The positive $\mathrm{Fe}(\mathrm{III}) / \mathrm{Fe}(\mathrm{II})$ redox potential leads to high ORR activity ${ }^{26}$; therefore, measurement of the $\mathrm{Fe}(\mathrm{III}) / \mathrm{Fe}(\mathrm{II})$ redox potential is significant to determine the catalytic activity of the prepared electrodes. To evaluate the redox potential of the catalysts, cyclic voltammetric $(\mathrm{CV})$ curves of FePc-DMSO/oxMWCNT_20 wt\%, FeAzPc-4N-THF/ oxMWCNT_50 wt\%, and FeAzPc-4N-DMSO/oxMWC NT_20 wt\% were measured in $\mathrm{N}_{2}$-saturated $0.1 \mathrm{M} \mathrm{KOH}$ solutions (Fig. 5b). The redox peaks of FePc-DMSO/ oxMWCNT_20 wt\%, FeAzPc-4N-THF/oxMWCNT_50 wt $\%$, and FeAzPc-4N-DMSO/oxMWCNT_20 wt\% attributed to $\mathrm{Fe}(\mathrm{III}) /(\mathrm{II})$ redox reactions ${ }^{34}$ were observed at $0.80,0.90$, and $0.92 \mathrm{~V}$, respectively. The positive shift of the redox peak for FeAzPc-4N-DMSO/oxMWCNT_20 wt\% and FeAzPc4N-THF/oxMWCNT_50 wt\% relative to that for FePcDMSO/oxMWCNT_20 wt\% strongly supports the high ORR activities of these catalysts. The larger positive shift for FeAzPc-4N-DMSO/oxMWCNT_20 wt\% than for FeAzPc4N-THF/oxMWCNT_50 wt\% implies that molecularly adsorbed FeAzPc-4N has higher catalytic properties, which confirms the results of LSV measurements.

The amount of adsorbed FePc derivatives on oxMWCNTs was estimated from the quantity of electric charge at the $\mathrm{Fe}(\mathrm{III}) /(\mathrm{II})$ redox reaction peaks ${ }^{23}$. The 

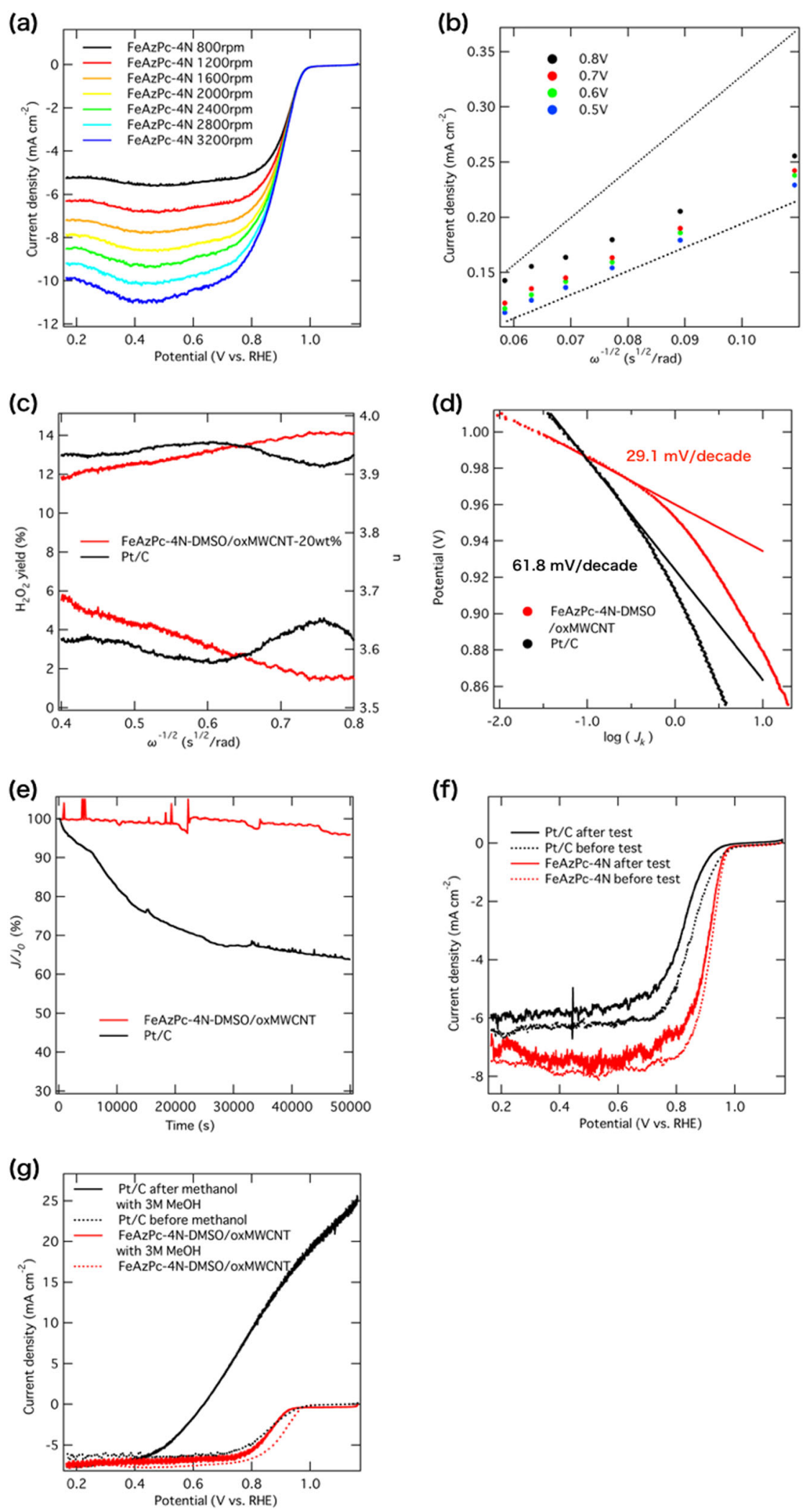

Fig. 4 (See legend on next page.) 
(see figure on previous page)

Fig. 4 ORR activities of FeAzPc-4N-DMSO/oxMWCNT_20 wt\%. a Oxygen reduction reaction polarization curves at different rotation rates in an $\mathrm{O}_{2}$-saturated $0.1 \mathrm{M} \mathrm{KOH}$ solution. b Koutecky-Levich plots calculated from the results in a. $\mathbf{c ~ H O}_{2}{ }^{-}$yield measured for Pt/C (black) and FeAzPc-4NDMSO/oxMWCNT_20 wt\% (red). d Tafel plots for Pt/C (black) and FeAzPc-4N-DMSO/oxMWCNT_20 wt\% (red). e Chronoamperometric test curves for $\mathrm{Pt} / \mathrm{C}$ (black) and FeAzPc-4N-DMSO/oxMWCNT_20 wt\% (red) with a given potential of $0.5 \mathrm{~V}$ vs. reversible hydrogen electrode in an $\mathrm{O}_{2}$-saturated $0.1 \mathrm{M}$ $\mathrm{KOH}$ solution at a rotation speed of $1600 \mathrm{rpm}$. $\mathbf{f}$ Linear sweep voltammetric (LSV) curves for Pt/C (black) and FeAzPc-4N-DMSO/oxMWCNT_20 wt\% (red) before (dashed) and after (solid) the chronoamperometric test. g LSV curves for Pt/C (black) and FeAzPc-4N-DMSO/oxMWCNT_20 wt\% (red) in $0.1 \mathrm{M} \mathrm{KOH}$ (dashed) and (solid) in $0.1 \mathrm{M} \mathrm{KOH}$ with $3.0 \mathrm{M}$ methanol
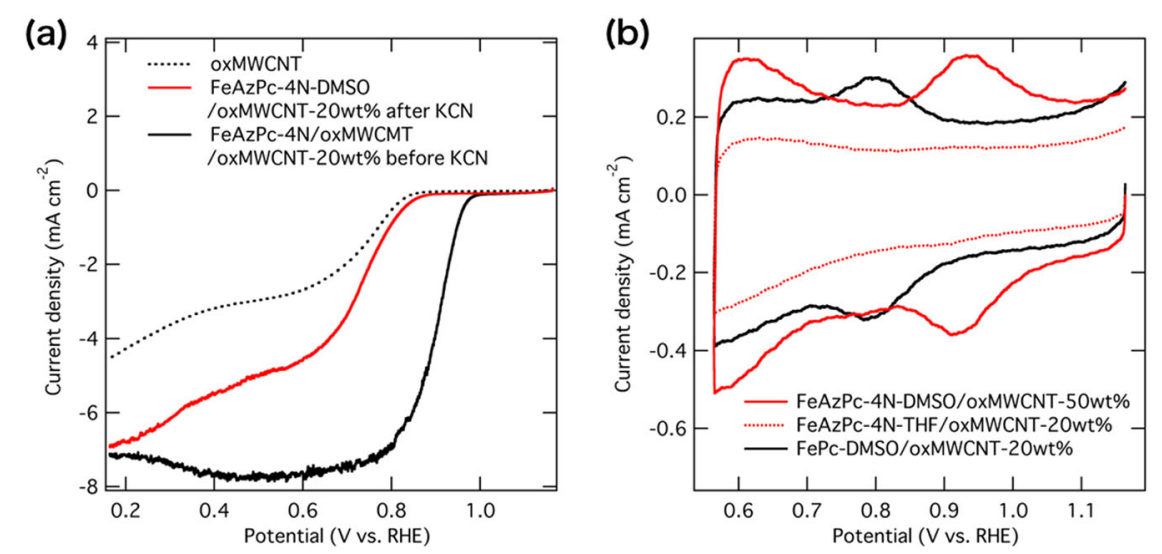

Fig. 5 a Effect of $\mathrm{CN}^{-}$poisoning of FeAzPc-4N-DMSO/oxMWCNT_20 wt\% measured in $\mathrm{O}_{2}$-saturated $0.1 \mathrm{M} \mathrm{KOH}$ before (black) and after (red) the addition of $10 \mathrm{mM} \mathrm{KCN}$. b Cyclic voltammetric curves for FePc-DMSO/oxMWCNT_20 wt\%, FeAzPc-4N-THF/oxMWCNT_50 wt\%, and FeAzPc-4NDMSO/oxMWCNT_20 wt\% measured in $\mathrm{N}_{2}$-saturated $0.1 \mathrm{M} \mathrm{KOH}$ solutions

concentration dependence of FeAzPc-4N on the amount of adsorbed molecules was also estimated, and the results are summarized in Table S1. It is noteworthy that more electroactive FeAzPc-4N is present at the surface of FeAzPc-4N-DMSO/oxMWCNT_20 wt\% than at the surface of FeAzPc-4N-THF/oxMWCNT_50 wt\%. The estimated amount of FeAzPc-4N adsorbed on oxMWCNTs increased with the mixing concentration of these molecules up to $20 \mathrm{wt} \%$. However, the number of adsorbed molecules decreased at $40 \mathrm{wt} \%$ compared with that at $20 \mathrm{wt} \%$. This decrease may be due to crystal formation and a decrease in the electronic conductivity of the catalytic electrodes due to overcoverage of the catalytic molecules on the oxMWCNTs. These results correspond well with the results obtained by LSV measurements.

TEM and XRD measurements indicated that FeAzPc$4 \mathrm{~N}$ molecules form crystals in the case of FeAzPc-4NTHF/oxMWCNT_50 wt\% and that those crystals have low electronic conductivity ${ }^{35}$. On the other hand, molecular layers of FeAzPc-4N are formed on the surface of FeAzPc-4N-DMSO/oxMWCNT_20 wt\%, which induces activation of the metal centers by electron withdrawing due to strong interaction between FeAzPc-4N and oxMWCNT $^{24}$. This is the reason why FeAzPc- $4 \mathrm{~N}$ DMSO/oxMWCNT_20 wt\% has higher ORR activity than FeAzPc-4N-THF/oxMWCNT_50 wt\%.

\section{Density functional theory (DFT) calculations}

To characterize the high ORR activities of FeAzPc-4N, DFT calculations of the ORR process on FePc and FeAzPc- $4 \mathrm{~N}$ were performed according to the methods shown in a previous report ${ }^{36}$. From the DFT calculations. $\Delta E_{\text {LUMO-HOMO }}(\mathrm{FePc})$ and $\Delta E_{\text {LUMO-HOMO }}($ FeAzPc-4N) were 1.88 and $2.04 \mathrm{eV}$, respectively. These band gap differences between FePc and FeAzPc-4N are in good agreement with those calculated from the results of UVVis adsorption. Mulliken charges of FePc and FeAzPc-4N before and after adsorption of an oxygen molecule were also calculated. Before adsorption, the Mulliken charges of Fe atoms of FePc and FeAzPc-4N were +0.801 and +0.819 , respectively. After adsorption of the oxygen molecule, the values increased to +1.001 and +1.008 , respectively. Note that the oxygen molecule selectively absorbed on the Fe atom since other complexes in which the oxygen molecule adsorbed on other atoms on FePc and FeAzPc-4N were not stable compared with the Feoxygen complex (see supporting information S12, and S13).

Figure 6 shows free-energy diagrams of ORR on FePc and FeAzPc-4N. From the four-electron $\left(4 e^{-}\right)$pathway at FePc (Fig. 6a), the maximum potential $\left(U_{\max }\right)$ was $0.20 \mathrm{~V}$, and the reaction proceeded successively from oxygen molecules to water via the downhill energy cascade. On the other hand, the energy becomes negative in the case of 

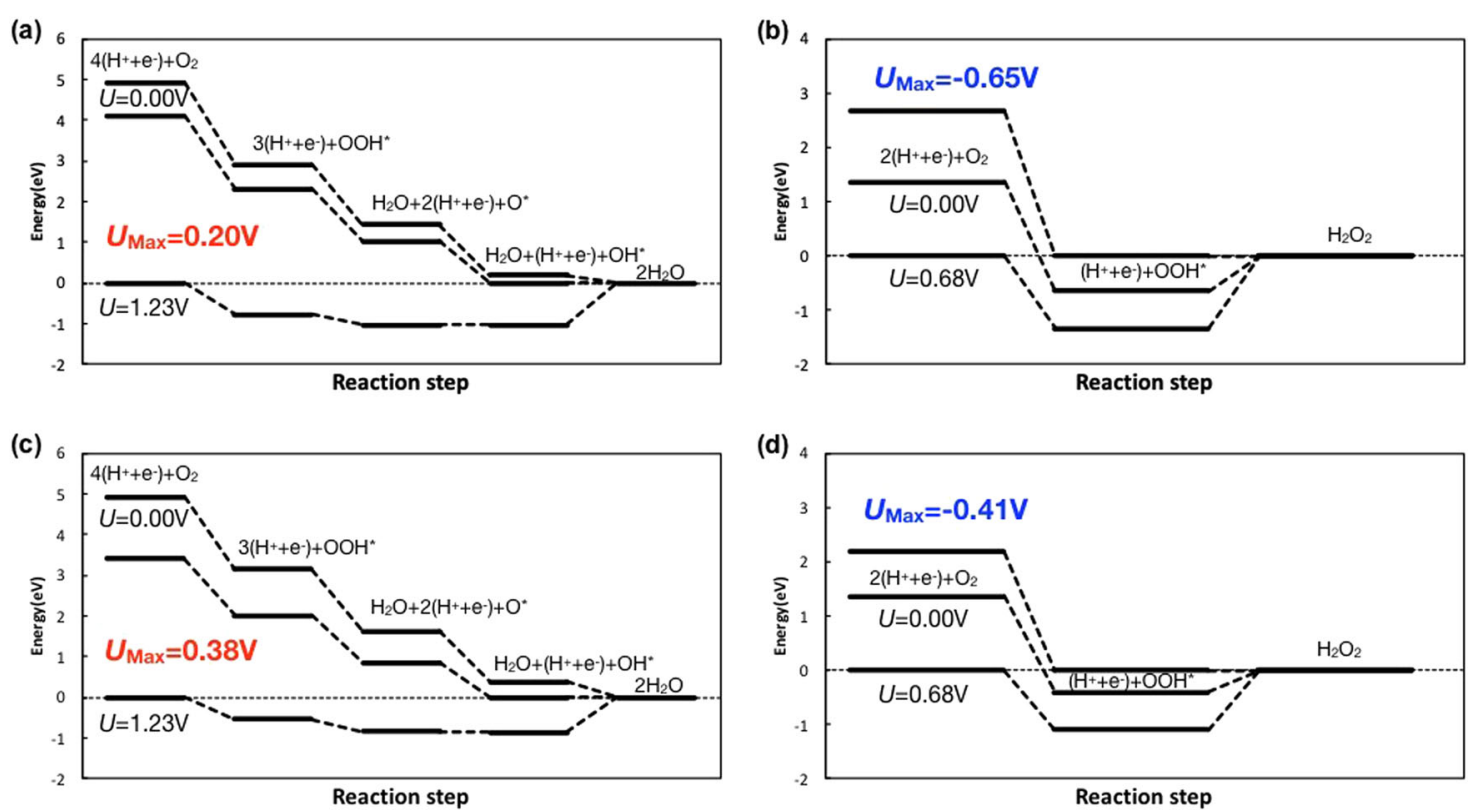

Fig. 6 Energy diagram of $4 e^{-}$and $2 e^{-}$oxygen reduction reaction pathways at FePc $(\mathbf{a}, \mathbf{b})$ and FeAzPc-4N (c, d), respectively

the two-electron $\left(2 e^{-}\right)$pathway, which indicates that the $2 e^{-}$process is negligible (Fig. 6b). Thus the ORR mainly occurred via the $4 e^{-}$pathway. In the case of FeAzPc-4N, the $2 e^{-}$pathway is also negligible (Fig. $6 \mathrm{~d}$ ), and the $4 e^{-}$ pathway also proceeded successfully (Fig. 6c). The $U_{\max }$ of the $4 e^{-}$pathway increased to $0.38 \mathrm{~V}$, which was caused by the higher free energy of $\mathrm{H}_{2} \mathrm{O}+\left(\mathrm{H}^{+}+e^{-}\right)+\mathrm{OH}^{*}$ of FeAzPc-4N than that of FePc. Since the difference between the $U_{\max }$ of FePc and that of FeAzPc-4N is directly related to the onset potential difference of ORR activities on those catalytic electrodes, these DFT results strongly support the high catalytic activities of FeAzPc$4 \mathrm{~N}$.

\section{Conclusions}

A new class of Pt-free catalysts composed of an Fe AzUL adsorbed on oxMWCNTs was designed. FeAzPcs could be dissolved in organic solvents such as DMSO, and catalytic electrodes modified with molecularly adsorbed FeAzPcs were successfully prepared. The optimized composition of the catalytic electrodes was determined, which resulted in superior ORR activities and durability relative to conventional FePc catalytic electrodes and commercial $\mathrm{Pt} / \mathrm{C}$ due to the electronwithdrawing properties of pyridinic nitrogen in the FeAzPcs. The catalytic electrodes molecularly modified with FeAzPcs have higher activities than those composed of FeAzPc crystals and oxMWCNTs. The high catalytic activity of FeAzPc was also proven using DFT calculations. To the best of our knowledge, these catalysts exhibit the highest activity among all conventional catalysts modified on MWCNTs and oxMWCNTs. The catalytic electrodes can be prepared at a lower cost than other Pt-free catalytic electrodes and without pyrolysis; therefore, these are promising catalytic electrode materials that are applicable to polymer electrolyte fuel cells and metal-air batteries.

\section{Experimental}

\section{Synthesis and characterization of FeAzPcs}

Scheme S1 shows the synthesis of FeAzPc-4N. 2,3Dicyanopyridine (258 mg, TCI), $\mathrm{FeCl}_{3} \bullet 6 \mathrm{H}_{2} \mathrm{O}$ (158 mg, Wako), and DBU (20 mg, TCI) were dissolved in a mixture of methanol (5 mL, Wako) and DMSO (5 mL, Wako). The solution was heated at $170{ }^{\circ} \mathrm{C}$ for $4 \mathrm{~h}$ with stirring and $\mathrm{N}_{2}$ bubbling. The color of the solution changed from orange to dark green. Then $5 \mathrm{~mL}$ of $1 \mathrm{M} \mathrm{HCl}$ aq. was added to degrade the intermediates. The products were collected by centrifugation at $6300 \mathrm{rpm}$ for $5 \mathrm{~min}$. The products were then dissolved in concentrated sulfuric acid and dropped into water to purify the products. Finally, the purified products were collected by centrifugation and dried in a vacuum oven at room temperature.

The final products were characterized using ESI-MS (LTQ Orbitrap Discovery, Thermo Fisher Scientific, USA) and UV-Vis spectroscopy (V760DS, JASCO, Japan). To determine the solubility of FePc and FeAzPc-4N, these products were dissolved in DMSO, DMF, and THF and centrifuged at $6300 \mathrm{rpm}$ for $10 \mathrm{~min}$, and the absorbance of the supernatants was measured. The saturated concentrations were calculated from absorbanceconcentration curves. 


\section{Modification of CNTs with FePc and FeAzPc}

First, $30 \mathrm{mg}$ of oxMWCNTs (No. 755125, Sigma Aldrich, USA) was dispersed in a DMSO solution of $0.1 \mathrm{mg} / \mathrm{mL}$ FeAzPc- $4 \mathrm{~N}$. The dispersion was sonicated with a homogenizer for $5 \mathrm{~min}$ and then suction-filtered to collect the samples. The samples were washed three times each with methanol and chloroform and then dried under vacuum. In the same way, $10 \mathrm{mg}$ of oxMWCNTs was added to $1 \mathrm{~mL}$ of a solution of FeAzPc-4N or FePc in THF $(1.0 \mathrm{mg} / \mathrm{mL})$ to prepare catalysts, which are designated as FeAzPc-4N-THF/oxMWCNT and FePc-THF/ oxMWCNT, respectively. FeAzPc-4N modified on MWCNT (No. 724769, Sigma Aldrich, USA) was prepared by the same process used for the FeAzPc-4N/ oxMWCNT samples.

\section{Characterization of catalysts}

The amount of FeAzPcs adsorbed on the CNTs was determined using XPS (PHI-5600, ULVAC-PHI, Inc., Japan). The amount of FeAzPc-4N on the oxMWCNTs was also determined by TG-DTA (TG8120, Rigaku, Japan) under an air atmosphere. The presence of FeAzPc crystals in the samples was confirmed by XRD (SmartLab 9MTP, RIGAKU, Japan) measurements.

\section{Microscopic observation of FeAzPc/oxMWCNTs}

The microstructure of the catalysts was observed by field emission scanning electron microscopy (FESEM; S5200, Hitachi Hi-Technologies Corp., Japan), STEM, and TEM (H-7650, Hitachi Hi-Technologies Corp., Japan), and the FESEM (Fig. S7) images of FeAzPc-4N-DMSO/ oxMWCNT revealed no aggregates in the sample. These results indicate that FeAzPc- $4 \mathrm{~N}$ molecules were adsorbed onto the oxMWCNTs.

The adsorption of FeAzPc-4N on the oxMWCNTs was investigated by STEM (JEM-ARM200F, JEOL Corp., Japan) equipped with a Cold Field Emission Gun and Cs probe corrector. STEM was performed at $80 \mathrm{kV}$. EELS spectra were acquired in STEM mode by a Gatan GIF Quantum ER spectrometer.

\section{Electrochemical measurements}

The ORR performance was evaluated by LSV and CV measurements using a potentiostat (2325, BAS Co., Ltd, Japan). Catalyst inks for each sample were prepared by dispersing $0.82 \mathrm{mg}$ of catalyst in a $1-\mathrm{mL}$ solution consisting of $6 \mu \mathrm{L}$ Nafion $^{\circledR}$ (527084, Sigma Aldrich, USA), $334 \mu \mathrm{L}$ isopropyl alcohol, and $84 \mu \mathrm{L}$ water by sonication for $5 \mathrm{~min}$. Then $20 \mu \mathrm{L}$ of the ink was cast onto a glassy carbon (GC, BAS Co., Ltd, Japan) of a RRDE (4 mm diameter, BAS Co., Ltd, Japan) and dried. The weight of the catalyst on the electrode was $307 \mu \mathrm{g} / \mathrm{cm}^{2}$. A Pt wire and a $\mathrm{Ag} / \mathrm{AgCl}$ electrode were inserted into the electrolyte as reference and counter electrodes, respectively. In all,
$0.1 \mathrm{M} \mathrm{KOH}$ solution bubbled with $\mathrm{N}_{2}$ or $\mathrm{O}_{2}$ for $30 \mathrm{~min}$ was used as the electrolyte. In addition, $3 \mathrm{M}$ methanol was added to an $\mathrm{O}_{2}$-saturated $0.1 \mathrm{M} \mathrm{KOH}$ solution for evaluation of the methanol oxidation activity.

The potential vs. $\mathrm{Ag} / \mathrm{AgCl}$ was converted to the $\mathrm{RHE}$ scale using the following equation:

$$
E(v s . R H E)=E(v s . A g / A g C l)+0.197+0.059 V \times p H
$$

The number of electrons $(n)$ involved in the ORR was calculated according to the K-L equation:

$$
\frac{1}{J}=\frac{1}{J_{k}}+\frac{1}{J_{d}}=\frac{1}{n F A k C_{O_{2}}}+\frac{1}{0.62 n F A D_{O_{2}}^{2 / 3} v^{-1 / 6} C_{O_{2}} \omega^{1 / 2}},
$$

where $J, J_{\mathrm{k}}$, and $J_{\mathrm{d}}$ are the measured kinetic and diffusion-limiting current, respectively; $F$ is the Faraday constant $(96,485 \mathrm{C} / \mathrm{mol}) ; A$ is the electrode area $\left(0.1256 \mathrm{~cm}^{2}\right) ; k$ is the rate constant for oxygen reduction $(\mathrm{m} / \mathrm{s}) ; D_{\mathrm{O} 2}$ is the diffusion coefficient of $\mathrm{O}_{2}$ in the electrolyte $\left(1.93 \times 10-5 \mathrm{~cm}^{2} / \mathrm{s}\right) ; v$ is the viscosity of the electrolyte solution $\left(1.009 \times 10^{-5} \mathrm{~cm}^{2} / \mathrm{s}\right) ; C_{\mathrm{O} 2}$ is the saturated concentration of $\mathrm{O}_{2}$ in the electrolyte $\left(1.26 \times 10^{-6} \mathrm{~mol} / \mathrm{cm}\right)$; and $\omega$ is the angular rotation rate. $n$ was also calculated using the RRDE results and the following equation:

$$
n=\frac{4 I_{D}}{\left(I_{D}+I_{R} / N\right)},
$$

where $J_{\mathrm{D}}$ and $J_{\mathrm{R}}$ are the current densities of disk and ring electrodes, respectively, and $N$ is the capture efficiency (0.42). The $\mathrm{H}_{2} \mathrm{O}_{2}$ yield can be determined by the following equation:

$$
\mathrm{H}_{2} \mathrm{O}_{2}(\%)=\frac{2 \times J_{R}}{\left(\mathrm{~N} \times\left|J_{D}\right|\right) J_{R}} \times 100
$$

\section{DFT calculations}

Theoretical calculations were carried out with DFT by using the Gaussian $09^{37}$ software package using the B3LYP hybrid functional ${ }^{38,39}$ with basis sets $6-31 G(d, p)$ for $\mathrm{C}, \mathrm{N}$, and $\mathrm{H}$ and cc-pVTZ for Fe. The optimum structure, partial charge (Mulliken charge) distribution, and energy levels of the highest occupied molecular orbital (HOMO) and the lowest unoccupied molecular orbital (LUMO) were calculated for two kinds of single molecules, FePc and FeAzPc-4N. DFT simulation of the ORR process on each catalytic electrode was performed via a method based on the computational hydrogen electrode model reported by Nørskov et al. ${ }^{40}$. Other simulations, including reaction pathways and free-energy diagrams of the ORR process, were performed according to a previous report ${ }^{36}$.

\section{Acknowledgements}

This work was partially supported by Kakenhi Grants-in-Aid (Nos. 17H01223, 18H05482, and 19K15598) from the Japan Society for the Promotion of Science (JSPS) and a Grant-in-Aid for JSPS Fellows from JSPS (No. 16J02105). This work was 
also partially supported by the WPI-AIMR fusion research program under the World Premier International Research Center Initiative (WPI), the Ministry of Education, Culture, Sports, Science and Technology, Japan (MEXT). Y.M., H.A., and H.Y. would like to express their gratitude to Mrs. Naomi Hirai of Hokkaido University for technical assistance with HAADF-EELS and Nanotechnology platform support. H.A., Y.H., and H.Y. would like to thank Mr. Nozaki Kohei of Tohoku University for support with electrochemical measurements and structural analyses. H.A. would like to thank Mr. Koji Yokoyama of Tohoku University for very helpful advice regarding electrochemical measurements. Mass spectrometry was partially supported by IA division, GFC, Hokkaido University.

\section{Author details}

'WPI-Advanced Institute for Materials Research, Tohoku University, 2-1-1, Katahira, Aoba-ku, Sendai 980-8577, Japan. ${ }^{2}$ Frontier Research Institute for Interdisciplinary Sciences, Tohoku University, 6-3, Aramaki-aza Aoba, Aoba-ku, Sendai 980-8578, Japan. ${ }^{3}$ Graduate School of Engineering, Tohoku University, 6-6, Aramaki-Aza-Aoba, Aoba-ku, Sendai 980-8579, Japan. ${ }^{4}$ Research Institute for Electronic Science (RIES), Hokkaido University, Kita21, Nishi10, Kita-Ku, Sapporo 001-0021, Japan. ${ }^{5}$ Department of Engineering Science, The University of Electro-Communications (UEC-Tokyo), 1-5-1 Chofugaoka, Chofu, Tokyo 182-8585, Japan. ${ }^{6}$ Graduate School of Environmental Studies, Tohoku University, 6-6-11-604 Aramaki-aza Aoba, Aoba-ku, Sendai 980-8579, Japan

\section{Authors' contributions}

H.A. and Y.H. wrote the manuscript. H.A. conceived the idea for this study, evaluated the catalytic activities by electrochemical measurements, and examined the catalytic activity mechanism. Y.H. synthesized FeAzPc molecules, modified CNTs with FeAzPcs, and conducted structural analysis of the catalysts using UV-Vis, TGA, and STEM. S.I. simulated the energy levels of the catalysts. H. M. and J.N. performed DFT calculations of the molecules. Y.M. and H.A. characterized the catalysts using ESI-MS and HAADF-EELS. H.A., T.M., and H.Y. designed the methodology to reach the conclusion. All other authors have contributed to data collection and interpretation and critically reviewed the manuscript. All authors approved the final version of the manuscript and agree to be accountable for all aspects of the work in ensuring that questions related to the accuracy or integrity of any part of the work are appropriately investigated and resolved.

\section{Conflict of interest}

The authors declare that they have no conflict of interest.

\section{Publisher's note}

Springer Nature remains neutral with regard to jurisdictional claims in published maps and institutional affiliations.

Supplementary information is available for this paper at https://doi.org/ 10.1038/s41427-019-0154-6.

Received: 22 May 2019 Revised: 4 July 2019 Accepted: 7 July 2019. Published online: 18 October 2019

\section{References}

1. Katsounaros, I., Cherevko, S., Zeradjanin, A. R. \& Mayrhofer, K. J. J. Oxygen electrochemistry as a cornerstone for sustainable energy conversion. Angew. Chem. Int. Ed. 53, 102-121 (2014).

2. Debe, M. K. Electrocatalyst approaches and challenges for automotive fuel cells. Nature 486, 43-51 (2012).

3. Cao, R., Lee, J.-S., Liu, M. \& Cho, J. Recent progress in non-precious catalysts for metal-air batteries. Adv. Energy Mater. 2, 816-829 (2012).

4. Pei, P., Wang, K. \& Ma, Z. Technologies for extending zinc-air battery's cyclelife: a review. Appl. Energy 128, 315-324 (2014).

5. Gasteiger, H. A. \& Marković, N. M. Just a dream—or future reality? Science $\mathbf{3 2 4}$ 48-49 (2009)

6. Sealy, C. The problem with platinum. Mater. Today 11, 65-68 (2008).

7. Liu, L. et al. Seaweed-derived route to Fe2O3 hollow nanoparticles/N-doped graphene aerogels with high lithium ion storage performance. ACS Appl. Mater. Interfaces 8, 7047-7053 (2016).
8. Yu, Y. M. et al. High active hollow nitrogen-doped carbon microspheres for oxygen reduction in alkaline media. Fuel Cells 12, 506-510 (2012).

9. Kong, J., Seyed Shahabadi, S. I. \& Lu, X. Integration of inorganic nanostructures with polydopamine-derived carbon: tunable morphologies and versatile applications. Nanoscale 8, 1770-1788 (2016).

10. Ozaki, J-i, Anahara, T., Kimura, N. \& Oya, A. Simultaneous doping of boron and nitrogen into a carbon to enhance its oxygen reduction activity in proton exchange membrane fuel cells. Carbon 44, 3358-3361 (2006).

11. Zhong, H. et al. ZIF-8 derived graphene-based nitrogen-doped porous carbon sheets as highly efficient and durable oxygen reduction electrocatalysts. Angew. Chem. Int. Ed. 53, 14235-14239 (2014).

12. Higgins, D., Zamani, P., Yu, A. \& Chen, Z. The application of graphene and its composites in oxygen reduction electrocatalysis: a perspective and review of recent progress. Energy Environ. Sci. 9, 357-390 (2016).

13. Abe, H., Nozaki, K., Kumatani, A., Matsue, T. \& Yabu, H.N- and Fe-containing carbon films prepared by calcination of polydopamine composites selfassembled at air/water interface for oxygen reduction reaction. Chem. Lett. 2, cl.180872 (2018)

14. Jasinski, R. A new fuel cell cathode catalyst. Nature 201, 1212-1213 (1964).

15. Zagal, J. H. Metallophthalocyanines as catalysts in electrochemical reactions. Coord. Chem. Rev. 119, 89-136 (1992).

16. Lee, D. H., Lee, W. J., Lee, W. J., Kim, S. O. \& Kim, Y. H. Theory, synthesis, and oxygen reduction catalysis of Fe-porphyrin-like carbon nanotube. Phys. Rev. Lett. 106, 8-11 (2011)

17. Han, $Y$. et al. Electronic structure engineering to boost oxygen reduction activity by controlling the coordination of the central metal. Energy Environ. Sci. 11, 2348-2352 (2018)

18. Tsukihara, T. et al. Structures of metal sites of oxidized bovine heart cytochrome c oxidase at 2.8 A. Science 269, 1069-1074 (1995).

19. Tenhunen, R., Marver, H. S. \& Schmid, R. The enzymatic conversion of heme to bilirubin by microsomal heme oxygenase. Proc. Natl Acad. Sci. USA 61, 748-755 (1968).

20. Zhang, W., Lai, W. \& Cao, R. Energy-related small molecule activation reactions: oxygen reduction and hydrogen and oxygen evolution reactions catalyzed by porphyrin- and corrole-based systems. Chem. Rev. 117, 3717-3797 (2017).

21. Sawaguchi, T., Matsue, T., Itaya, K. \& Uchida, I. Electrochemical catalytic reduction of molecular oxygen by iron porphyrin ion-complex modified electrode. Electrochim. Acta 36, 703-708 (1991).

22. Cao, R. et al. Promotion of oxygen reduction by a bio-inspired tethered iron phthalocyanine carbon nanotube-based catalyst. Nat. Commun. 4 1-7 (2013).

23. Yang, J., Toshimitsu, F., Yang, Z., Fujigaya, T. \& Nakashima, N. Pristine carbon nanotube/iron phthalocyanine hybrids with a well-defined nanostructure show excellent efficiency and durability for the oxygen reduction reaction. J. Mater. Chem. A 5, 1184-1191 (2017).

24. Jiang, Y. et al. Enhanced catalytic performance of Pt-free iron phthalocyanine by graphene support for efficient oxygen reduction reaction. ACS Catal. $\mathbf{3}$, 1263-1271 (2013).

25. Hirai, Y., Sawano, B., Takaki, T. \& Yabu, H. A fluorescence indicator at extreme low $\mathrm{pH}$ region based on dissolution of $\mathrm{Zn}$ tetra-2,3-pyridoporphyradine (TPP) nanocrystal suspension. J. Nanosci. Nanotechnol. 18, 455-458 (2018).

26. Zagal, J. H. \& Koper, M. T. M. Reactivity descriptors for the activity of molecular MN4 catalysts for the oxygen reduction reaction. Angew. Chem. Int. Ed. 55, 14510-14521 (2016).

27. Hirai, Y., Sawano, B., Takaki, T., Matsuo, Y. \& Yabu, H. Metal phthalocyanine derivative nanocrystals: color-controlled and transparent dispersions by a onepot UV-assisted synthetic process. Chem. Lett. 46, 695-698 (2017).

28. Sedona, F. et al. Tuning the catalytic activity of Ag(110)-supported Fe phthalocyanine in the oxygen reduction reaction. Nat. Mater. 11, 970-977 (2012).

29. Liang, Y. et al. Co3O4 nanocrystals on graphene as a synergistic catalyst for oxygen reduction reaction. Nat. Mater. 10, 780-786 (2011).

30. Kattel, S. \& Wang, G. Reaction pathway for oxygen reduction on FeN4 embedded graphene. J. Phys. Chem. Lett. 5, 452-456 (2014).

31. Guo, D. et al. Active sites of nitrogen-doped carbon materials for oxygen reduction reaction clarified using model catalysts. Science 351, 361-365 (2016).

32. Thorum, M. S., Hankett, J. M. \& Gewirth, A. A. Poisoning the oxygen reduction reaction on carbon-supported Fe and Cu electrocatalysts: evidence for metalcentered activity. J. Phys. Chem. Lett. 2, 295-298 (2011). 
33. von Deak, D., Singh, D., King, J. C. \& Ozkan, U. S. Use of carbon monoxide and cyanide to probe the active sites on nitrogen-doped carbon catalysts for oxygen reduction. Appl. Catal. B Environ. 113-114, 126-133 (2012).

34. Dantas, L. M. F. et al. Electrocatalytic oxidation of hydrazine in alkaline media promoted by iron tetrapyridinoporphyrazine adsorbed on graphite surface. J. Braz. Chem. Soc. 19, 720-726 (2008).

35. Khrishnakumar, K. P. \& Menon, C. S. Determination of the thermal activation energy and grain size of iron phthalocyanine thin films. Mater. Lett. 48, 64-73 (2001).

36. Matsuyama, H., Akaishi, A. \& Nakamura, J. Effect of water on the manifestation of the reaction selectivity of nitrogen-doped graphene nanoclusters toward oxygen reduction reaction. ACS Omega 4, 3832-3838 (2019).

37. Frisch, M. et al. Gaussian 09, Revision D.01 (Gaussian Inc., 2009).

38. Lee, C., Hill, C. \& Carolina, N. Development of the Colle-Salvetti correlationenergy formula into a functional of the electron density. Chem. Phys. Lett. 162 165-169 (1989).

39. Becke, A. D. Density-functional thermochemistry. III. The role of exact exchange. J. Chem. Phys. 98, 5648-5652 (1993).

40. Nørskov, J. K. et al. Origin of the overpotential for oxygen reduction at a fuelcell cathode. J. Phys. Chem. B 108, 17886-17892 (2004). 